Family Medicine and Community Health

\title{
Case-based audit and feedback around a decision aid improved antibiotic choice and duration for uncomplicated cystitis in primary care clinics
}

\author{
Larissa Grigoryan, ${ }^{1}$ Roger Zoorob, ${ }_{1}^{1}$ George Germanos, ${ }^{1,2}$ Mohamad Sidani, ${ }^{1}$ \\ Matthew Horsfield, ${ }^{1}$ Fareed Khan, ${ }^{1}$ Mohammad Zare, ${ }^{3}$ Melanie Goebel, ${ }^{4}$ \\ Robert Atmar, ${ }^{4}$ Barbara Trautner ${ }^{5}$
}

To cite: Grigoryan L, Zoorob R, Germanos G, et al. Casebased audit and feedback around a decision aid improved antibiotic choice and duration for uncomplicated cystitis in primary care clinics. Fam Med Com Health 2021:9:e000834. doi:10.1136/fmch-2020-000834

Check for updates

(c) Author(s) (or their employer(s)) 2021. Re-use permitted under CC BY-NC. No commercial re-use. See rights and permissions. Published by BMJ.

${ }^{1}$ Family and Community Medicine, Baylor College of Medicine, Houston, Texas, USA ${ }^{2}$ Internal Medicine, Desert Regional Medical Center, Palm Springs, California, USA

${ }^{3}$ Family and Community Medicine, University of Texas McGovern Medical School, Houston, Texas, USA

${ }^{4}$ Section of Infectious Diseases, Department of Medicine, Baylor College of Medicine, Houston, Texas, USA

${ }^{5}$ Center for Innovations in Quality, Effectiveness and Safety, Michael E. DeBakey Veterans Affairs Medical Center and Department of Medicine, Baylor College of Medicine, Michael E DeBakey VA Medical Center, Houston, Texas, USA

Correspondence to Dr Larissa Grigoryan; grigorya@bcm.edu

\section{ABSTRACT}

Objectives The objective of our study was to evaluate the impact of a multifaceted stewardship intervention on adherence to the evidence-based practice guidelines on treatment of uncomplicated cystitis in primary care. We hypothesised that our intervention would increase guideline adherence in terms of antibiotic choice and duration of treatment.

Design A preintervention and postintervention comparison with a contemporaneous control group was performed. During the first two study periods, we obtained baseline data and performed interviews exploring provider prescribing decisions for cystitis at both clinics. During the third period in the intervention clinic only, the intervention included a didactic lecture, a decision algorithm and audit and feedback. We used a difference-in-differences analysis to determine the effects of our intervention on the outcome and guideline adherence to antibiotic choice and duration.

Setting Two family medicine clinics (one intervention and one control) were included.

Participants All female patients with uncomplicated cystitis attending the study clinics between 2016 and 2019.

Results Our sample included 932 visits representing 812 unique patients with uncomplicated cystitis. The proportion of guideline-adherent antibiotic regimens increased during the intervention period (from $33.2 \%$ (95\% Cl 26.9 to 39.9 ) to $66.9 \%(95 \% \mathrm{Cl} 58.4$ to 74.6$)$ in the intervention site and from $5.3 \%(95 \% \mathrm{Cl} 2.3$ to 10.1$)$ to $17.0 \%(95 \% \mathrm{Cl}$ 9.9 to 26.6$)$ in the control site). The increase in guideline adherence was greater in the intervention site compared with the control site with a difference-in-differences of 22 percentage points, $p=0.001$.

Conclusion A multifaceted intervention increased guideline adherence for antibiotic choice and duration in greater magnitude than similar trends at the control site. Future research is needed to facilitate scale-up and sustainability of case-based audit and feedback interventions in primary care.

\section{INTRODUCTION}

Antimicrobial resistance is a well-recognised threat to global health, with the USA alone

\section{Key points}

\section{Question}

- We evaluated the impact of audit and feedback antibiotic stewardship intervention on guideline adherence for antibiotic choice and duration for acute uncomplicated cystitis in primary care.

\section{Finding}

- Our multifaceted intervention increased guideline adherence for antibiotic choice and duration in greater magnitude than similar trends at the control site. Using the difference-in-differences design, we demonstrated that a case-based audit and feedback intervention can increase the proportion of primary care clinic visits for urinary tract infections in which women receive the right drug with the right duration, a fundamental aspect of antibiotic stewardship.

\section{Meaning}

- Our study added evidence to the limited literature regarding antimicrobial stewardship interventions for cystitis in the outpatient setting, a neglected practice area in the US antibiotic stewardship programmes. Future research will focus on scale-up and sustainability of case-based audit and feedback interventions in primary care.

accounting for 2.8 million antibiotic-resistant infections and 35000 deaths each year. ${ }^{1}$ Increasing realisation of the need to minimise this public health threat can be seen in the efforts of regulatory bodies such as the Joint Commission, which recently established requirements for antimicrobial stewardship for ambulatory healthcare organisations, effective at the beginning of $2020 .^{2}$ The new requirements mandate that such organisations provide resources to practitioners to promote appropriate antibiotic prescribing practices. $^{2}$

Although most studies of outpatients have focused on implementing antibiotic 
stewardship for upper respiratory infections, ${ }^{34}$ there is also a high prevalence of inappropriate antibiotic prescribing for urinary tract infections (UTIs) in primary care, including overuse of fluoroquinolones and longer duration of treatment than recommended by guidelines. ${ }^{5-10} \mathrm{~A}$ study in France found that only $20 \%$ of outpatient UTIs were treated with the guidelines-recommended drug, dose and duration. ${ }^{11}$ An Irish study found that only $55 \%$ of the antibiotic prescriptions for UTI in general practice were appropriate. ${ }^{12}$ Of 7738 outpatient encounters for UTI in Israel, $91 \%$ were treated with a longer duration of antibiotics than recommended by guidelines. ${ }^{13} \mathrm{~A}$ recent US study revealed that fluoroquinolones were the most commonly prescribed antibiotics for uncomplicated UTI, comprising up to $49 \%$ of prescriptions. ${ }^{6}$ Inappropriate use of fluoroquinolones is especially concerning because it promotes the emergence and spread of multidrugresistant Escherichia coli strain sequence type $131 .{ }^{14}$ In addition, continued overprescribing of fluoroquinolones for uncomplicated cystitis in patients with other treatment options is occurring in the USA ${ }^{515}$ despite two black-box warnings from the US Food and Drug Administration (FDA) for fluoroquinolones due to an association between their use and serious side effects. ${ }^{16-18}$ In addition, fluoroquinolones were associated with more central nervous system-related and gastrointestinal-related adverse events compared with other types of antimicrobials in a recent meta-analysis. ${ }^{19}$ Excessive treatment duration for uncomplicated cystitis is another common problem documented internationally. ${ }^{913}$ Current Infectious Diseases Society of America (IDSA) guidelines ${ }^{20}$ recommend nitrofurantoin for 5 days, trimethoprim-sulfamethoxazole for 3 days and a single dose of fosfomycin as the first-line regimens for uncomplicated cystitis. In our previous study in the same setting, most prescriptions for trimethoprim-sulfamethoxazole, nitrofurantoin and fluoroquinolones had a treatment duration longer than recommended. ${ }^{9}$

Unlike upper respiratory infections, which typically involve viral infections for which antibiotics are not indicated, a symptomatic UTI merits treatment with antibiotics, as recommended by the IDSA guidelines. ${ }^{20}$ Thus, the focus in implementing antibiotic stewardship for UTI needs to be optimisation of antibiotic choice and duration, which may present a different cognitive challenge for practitioners than deciding whether a patient needs antibiotics or not. One evidence-based strategy shown to be effective in implementing an antimicrobial stewardship for UTI in acute and long-term care settings is audit and feedback. ${ }^{21-23}$ Multiple strategies have been employed for implementing stewardship such as feedback to prescribers on antimicrobial consumption and antimicrobial stewardship committee. ${ }^{24}$ Based on our prior successful experience with audit and feedback in acute and long-term care, ${ }^{21}$ we implemented a multifaceted antimicrobial stewardship intervention using audit and feedback to improve compliance with acute cystitis guidelines in a family medicine setting (general practice). The objective of our study was to evaluate the impact of
Table 1 Clinic and prescriber characteristics of intervention and control sites*

\begin{tabular}{|c|c|c|}
\hline & Intervention site & Control site \\
\hline $\begin{array}{l}\text { Number of } \\
\text { physicians }\end{array}$ & 9 & 6 \\
\hline $\begin{array}{l}\text { Board certified } \\
\text { in family } \\
\text { medicine }\end{array}$ & 9 & $5 \dagger$ \\
\hline $\begin{array}{l}\text { Number of } \\
\text { physician } \\
\text { assistants }\end{array}$ & 2 & 1 \\
\hline $\begin{array}{l}\text { Services } \\
\text { provided }\end{array}$ & $\begin{array}{l}\text { Preventive and acute } \\
\text { care } \\
\text { Behavioural health } \\
\text { Nutrition services } \\
\text { Onsite laboratories }\end{array}$ & $\begin{array}{l}\text { Preventive and } \\
\text { acute care } \\
\text { Behavioural health } \\
\text { Nutrition services } \\
\text { Onsite laboratories }\end{array}$ \\
\hline $\begin{array}{l}\text { UTI antibiotics } \\
\text { per } 1000 \text { office } \\
\text { visits }\end{array}$ & 20.4 & 30.4 \\
\hline
\end{tabular}

*Intervention and control sites are non-teaching clinics in a single academic medical centre; no clinical pharmacist in either clinic. †One physician was board certified in internal medicine. UTI, urinary tract infection.

a multifaceted stewardship intervention on adherence to the evidence-based practice guidelines on treatment of uncomplicated cystitis in primary care. We hypothesised that our intervention would increase guideline adherence in terms of antibiotic choice and duration of treatment.

\section{METHODS}

\section{Study design and settings}

We used a difference-in-differences study design to determine the effects of our stewardship intervention on adherence to the IDSA guidelines ${ }^{20}$ and recommendations from the American Academy of Family Physicians $(\mathrm{AAFP})^{25}$ for treating acute cystitis and measuring antibiotic choice and duration. ${ }^{20}$ A preintervention and postintervention comparison with a contemporaneous control group from July 2016 to March 2019 was performed at two private, academically affiliated family medicine clinics in a large urban area. We chose two clinics (intervention and control sites) within the same private US healthcare system because they were similar in terms of patient populations, provider type (predominantly physicians with two physician assistants at each clinic) and electronic medical record (EMR) software. Table 1 shows clinic and prescriber characteristics at the intervention and control sites. Both sites provide preventive and acute care, behavioural health, nutrition services and onsite laboratories. All physicians, except for one, are board certified in family medicine. On average, 3248 appointments for a cohort of 19777 patients occur at these clinics each month. Patients in both clinics are predominantly women $(58 \%)$ and Caucasian $(54 \%)$. 


\section{Study population}

The study population included all patients with acute uncomplicated cystitis at the intervention and control sites. Inclusion criteria for uncomplicated cystitis required that participants be women $\geq 18$ years who had International Classification of Diseases, Tenth Revision (ICD10) codes (N30.0, acute cystitis; N30.9, cystitis unspecified; and N39.0 UTI site not specified) for UTI listed as a diagnosis in the EMR system (Epic Clarity database). In addition to a UTI-related diagnosis, patients must have also been prescribed a UTI-relevant antibiotic during the same visit. UTI-relevant antibiotics included fluoroquinolones, nitrofurantoin, fosfomycin, trimethoprim alone or in combination with sulfamethoxazole, betalactams and aminoglycosides. The electronic algorithm, using ICD-10 UTI diagnosis codes paired with medication data to identify patients with UTI, was validated in the same setting. ${ }^{26}$ Visits that met criteria for complicated UTI or had recorded signs or symptoms of pyelonephritis were excluded (eg, an additional code for genitourinary abnormalities or recorded fever, defined as $\geq 100.4^{\circ} \mathrm{F}$ or $38^{\circ} \mathrm{C}$ ). We also excluded five patients who had allergies to both nitrofurantoin and sulfa-containing antibiotics and those prescribed long-term antibiotics indicating prophylaxis for recurrent UTI (figure 1).

For each eligible visit, we extracted the following variables: patient age, race, comorbidities (Charlson Comorbidity Score), antibiotic allergies, type of antibiotic prescribed and duration of treatment. If a patient returned to the clinic within 7 days of initial treatment due to UTI,

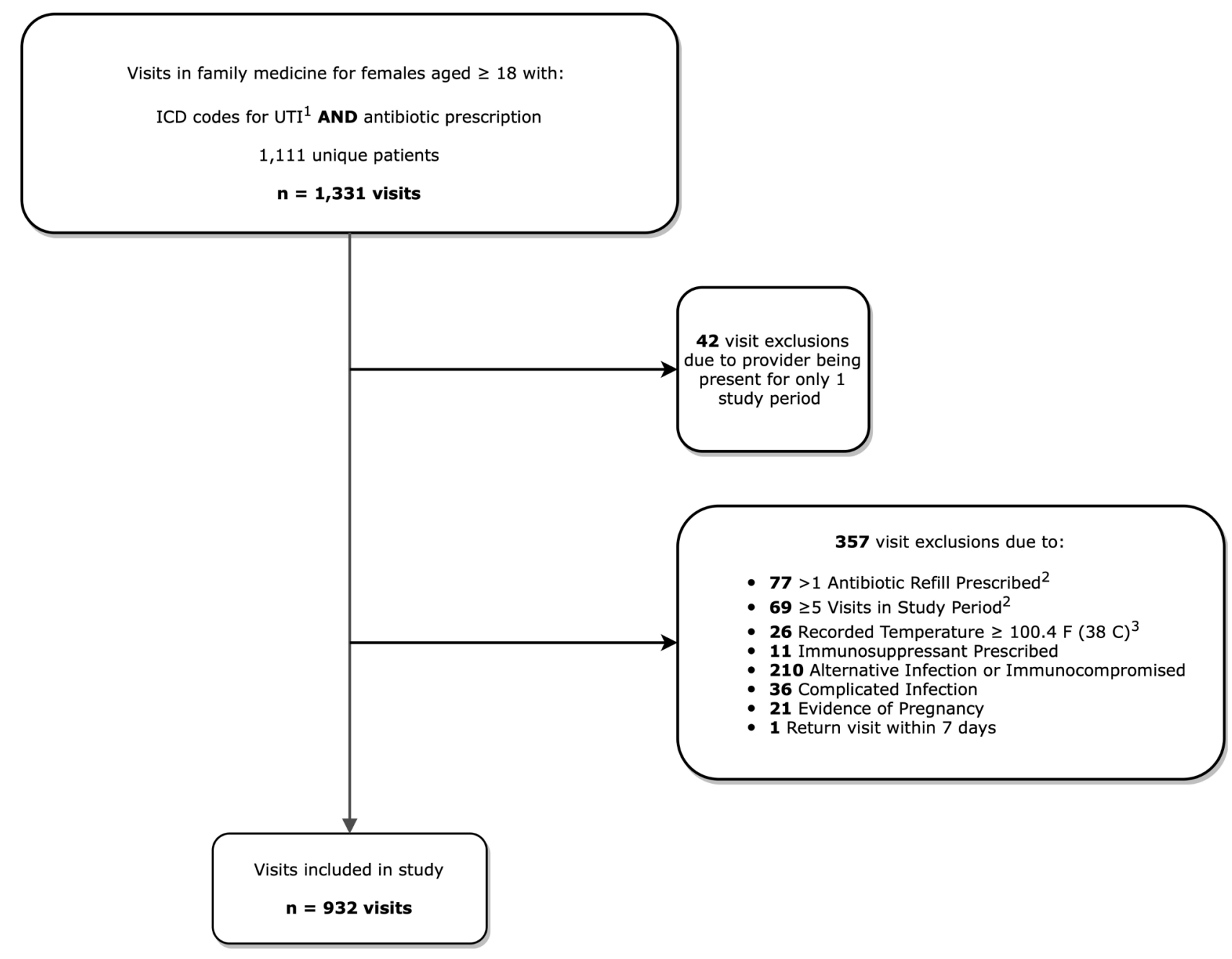

Footnote:

${ }^{1}$ ICD-10 codes N30.0 acute cystitis, N30.9 cystitis unspecified and N39.0 urinary tract infection site not specified

$2 \geq 5$ visits in the study period and $>1$ antibiotic refill indicate recurrent UTI

${ }^{3}$ Recorded temperature $>100.4 \mathrm{~F}(38 \mathrm{C}$ ) indicates pyelonephritis

Figure 1 Selection process used to determine uncomplicated cystitis visits in the study period*. The study period was from July 2016 to February 2019. *Visits may have had more than one exclusion criteria. UTI, urinary tract infection; ICD, International Classification of Diseases. 


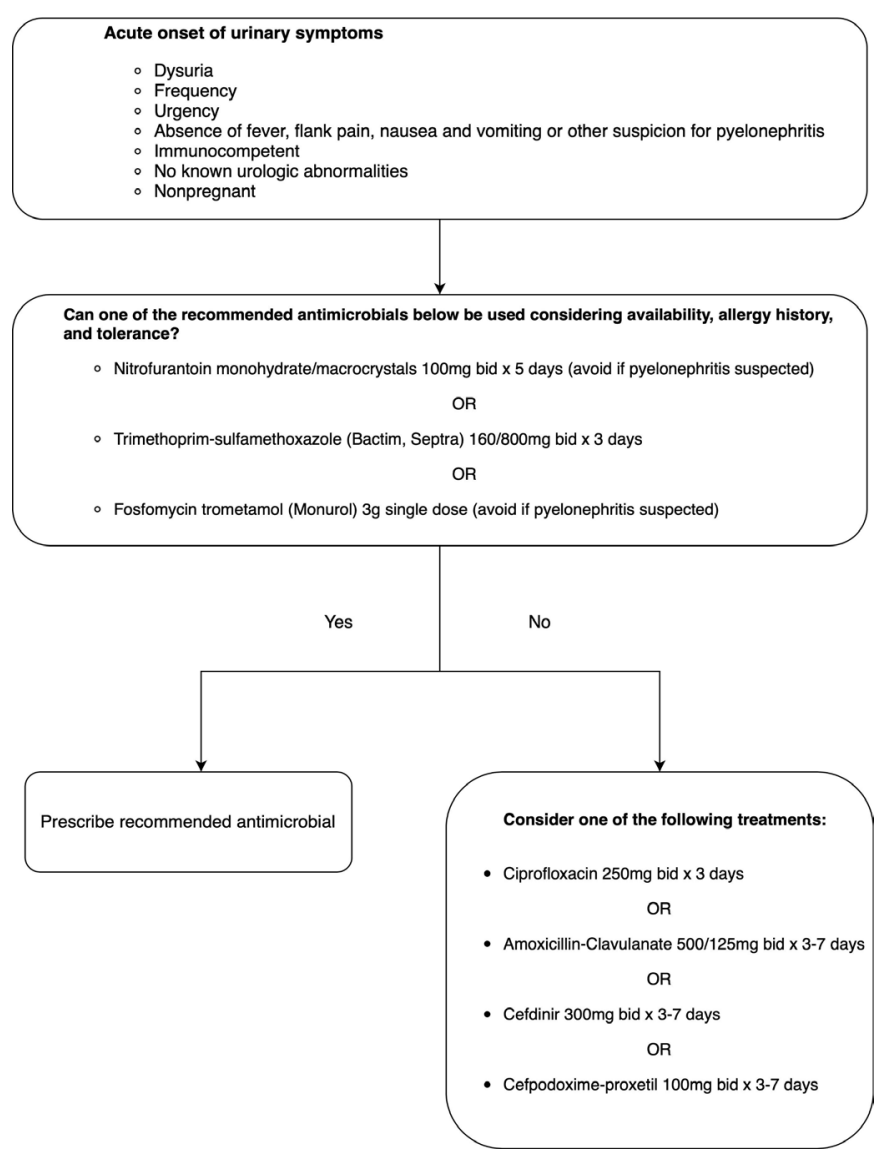

Figure 2 Pocket card on choosing empirical antibiotic treatment for acute cystitis based on Infectious Diseases Society of America guidelines. bid, two times per day.

the case was considered as a failure of previous treatment. In these cases, only the original visits were included in the study. We included women with diabetes because recent evidence suggests that diabetic women presenting with acute cystitis in primary care should be managed similarly to women without diabetes. ${ }^{27}$ We also included women aged $\geq 65$ years, as treatment recommendations for otherwise healthy older women are similar to those for younger women. ${ }^{20}$ Each record was manually reviewed by a team member (GG or MG) to rule out the possibility of contraindication to all first-line antibiotics. All cases of acute uncomplicated cystitis during the study period were included in the analysis.

\section{Outcome measure}

The outcome of this study was in adherence to the IDSA guidelines for managing uncomplicated cystitis, both to medication choice and duration of therapy (figure 2). For example, prescribing a guideline-adherent antibiotic (nitrofurantoin) for excessive duration ( 7 days) would be counted as non-adherent. Likewise, prescribing ciprofloxacin (a non-first-line antibiotic choice) for the correct duration (3 days) would be counted as non-adherent. Prescribing a first-line agent (trimethoprim-sulfamethoxazole) for the correct duration ( 3 days) would be counted as compliant. Likewise, prescribing nitrofurantoin for 5 days or a single dose of fosfomycin would be counted as compliant. ${ }^{20}$

\section{Intervention development}

All activities in the intervention and control sites during the study period are described in table 2 . In the first study period, we validated our electronic algorithm ${ }^{26}$ and obtained baseline data on the outcome. In the second study period, we interviewed providers to explore their prescribing decisions for UTI to help us understand why they were choosing certain drugs or durations of treatment. The findings from these interviews, published elsewhere, ${ }^{28}$ were used to develop educational materials (interactive case-based lecture) for the intervention. For example, we found that providers were misled by advanced patient age, diabetes and recurrent UTI to make inappropriate choices for acute cystitis. We therefore focused our teaching cases on these points, presenting actual cases of patients who had visited one of the clinics in the previous 2 months. Baseline period activities also

Table 2 List of activities at intervention and control sites

\begin{tabular}{|c|c|c|}
\hline Study period & Intervention site & Control site \\
\hline $\begin{array}{l}\text { Baseline } \\
\text { (July } 2016 \text { to } \\
\text { March 2017) }\end{array}$ & Baseline measurements of outcomes & $\begin{array}{l}\text { Baseline measurements of } \\
\text { outcomes }\end{array}$ \\
\hline $\begin{array}{l}\text { Interviews } \\
\text { (April } 2017 \text { to } \\
\text { March 2018) }\end{array}$ & $\begin{array}{l}\text { Interviews with providers about treatment of uncomplicated cystitis } \\
\text { Refine existing intervention materials based on interview results }\end{array}$ & $\begin{array}{l}\text { Interviews with providers about } \\
\text { treatment of uncomplicated } \\
\text { cystitis }\end{array}$ \\
\hline
\end{tabular}




\section{Box 1 Audit and feedback script (example)}

'Your patient (45 years old) presented with symptoms of urinary tract infection (UTI) (dysuria and urinary frequency), and you diagnosed her with UTI.

According to the guidelines, the first thing is to check whether the patient had any of the following symptoms of pyelonephritis: fever, flank pain, nausea and vomiting or other suspicion for pyelonephritis. Also, consider possible complicating factors such as urological abnormalities and immunocompromising conditions. Based on reviewing the chart, the patient didn't seem to have pyelonephritis or a complicating condition. Therefore, this was likely a case of acute uncomplicated cystitis. The patient did not have allergies to any of the first-line recommended antibiotics for UTI (trimethoprim-sulfamethoxazole, nitrofurantoin and fosfomycin). You decided to treat the patient empirically with nitrofurantoin for 7 days'.

\section{Feedback}

'Your choice of antibiotic fits with the guidelines. However, according to Infectious Diseases Society of America guidelines, nitrofurantoin can be prescribed for 5 days. Therefore, consider shortening your duration of treatment with nitrofurantoin to 5 days for future cases'.

included the development and validation of a search algorithm for identifying visits with UTI, as well as piloting of our decision aid (pocket card) (figure 2), and the audit and feedback intervention and script (box 1). We revised the existing audit and feedback script that we used in our previous successful intervention study ${ }^{21}$ in acute and long-term care to be used in the primary care setting.

The audit and feedback component of the intervention (based on feedback intervention theory) ${ }^{29} 30$ was a highly personalised, interactive, one-on-one intervention with primary care providers to improve their capacity to distinguish between uncomplicated cystitis and other UTI syndromes and to encourage them to prescribe a guideline-concordant antibiotic regimen. We also included information about first-line antibiotics recommended by the IDSA guidelines and AAFP and determined whether the antibiotic regimen prescribed by the providers was in accordance with the guidelines (box 1).

In the intervention period, we distributed guidelines at both sites (intervention and control). Distributing the guidelines addressed awareness, but we did not expect guideline dissemination alone to be an effective method to achieve behaviour change. ${ }^{31} 32$ At the intervention site, we also conducted a training session to help providers engage with and internalise guidelines content. Our educational session provided a detailed overview of the IDSA treatment guidelines; definitions for various UTI syndromes, including uncomplicated versus complicated UTI, and actual clinical examples. During our training session, we also taught the providers how to use the decision aid (figure 2). The investigators selected actual cases of UTI seen in the clinics to design both teaching cases to address the specific clinical scenarios that were problematic for the interview participants.

From April 2018 to February 2019, we performed an audit and feedback intervention, in which charts of women meeting study eligibility, as described in figure 1, were reviewed.

All cases of acute cystitis during the second phase of the study in the intervention clinic triggered a chart review. The patient's EMR was reviewed to determine the type of antibiotic prescribed and the duration of treatment. Appropriateness of the treatment was determined by the research team (LG, GG, MG and BT), using the IDSA guidelines. Our team included two infectious diseases doctors, an infectious diseases epidemiologist and a primary care research fellow. We randomly selected one case per provider per month to reduce the burden on providers, and the research team contacted each provider in person or by phone to provide follow-up as to whether the treatment decision was in compliance with IDSA guidelines. We built the script for our audit-feedback intervention using our previously published script used in acute and long-term care settings. ${ }^{21}$ The feedback was given to providers in person or by phone by the principal investigator (LG) within 5-7 days of the patient visit through postprescription antimicrobial review, using the algorithm. Feedback was given in both scenarios-when the prescribing was in accordance with the guidelines and when antibiotic choice and/or duration was not in accordance with the guidelines.

\section{Statistical analysis}

Sample size

In a previous US study, the adherence to cystitis management guidelines for antibiotic choice and treatment duration (our outcome) in the outpatient setting was $44 \%{ }^{22}$ We used this estimate and calculated the sample size of visits with cystitis needed at each of the two sites based on testing the differences in two independent proportions. We calculated that a total of 97 visits at each site would provide a power of $80 \%$ to detect an absolute difference of $20 \%$ in the postintervention rates between the two groups at a significance level of 0.05 .

We used $\chi^{2}$ test, Fisher exact test and $\mathrm{t}$ test to determine whether visit-level factors (age, race/ethnicity and Charlson Comorbidity Score) differed between the intervention and control sites. Difference-in-differences analysis was performed to determine intervention effectiveness using the composite outcome of guideline adherence in terms of antibiotic choice and duration of treatment. The difference-in-differences estimator is calculated by subtracting the change in proportion of guideline-adherent regimens between the intervention and baseline periods of the control site from the change in proportion of guideline-adherent regimens between the intervention and baseline periods of the intervention site.

We used log-binomial regression analyses for each outcome to calculate the relative risks (RRs) with $95 \%$ CIs and studied the interaction between study site (intervention and control) and study period (baseline, interviews and intervention). We specifically separated the baseline and interview periods because the interviews 
Table 3 Patient characteristics at intervention and control sites

\begin{tabular}{lccl}
\hline & $\begin{array}{l}\text { Intervention clinic } \\
\text { (n=546 visits) }\end{array}$ & $\begin{array}{l}\text { Control clinic } \\
\text { (n=386 visits) }\end{array}$ & P value* $^{*}$ \\
\hline $\begin{array}{l}\text { Mean age, } \\
\text { years }( \pm \text { SD) }\end{array}$ & $49.1 \pm 16.8$ & $46.8 \pm 15.9$ & $\mathbf{0 . 0 3}$ \\
\hline $\begin{array}{l}\text { Race/ethnicity } \\
\text { Caucasian }\end{array}$ & $307 / 491(62.5 \%)$ & $185 / 337(54.9 \%)$ & 0.06 \\
\hline $\begin{array}{l}\text { African } \\
\text { American }\end{array}$ & $115 / 491(23.4 \%)$ & $92 / 337(27.3 \%)$ & \\
Asian & $38 / 491(7.7 \%)$ & $41 / 337(12.2 \%)$ & \\
\hline Hispanic & $31 / 491(6.3 \%)$ & $19 / 337(5.6 \%)$ & 0.06 \\
$\begin{array}{l}\text { Charlson } \\
\text { Comorbidity }\end{array}$ & $82(15.0 \%)$ & $41(10.6 \%)$ & \\
Index $\geq 2$ & & & \\
\hline
\end{tabular}

${ }^{*} P$ values refer to $t$ test and $\chi^{2}$ tests.

†Race/ethnicity was missing for 104 visits.

may have affected providers' prescribing behaviour. The interaction term of these two variables was the differencein-differences estimator, and its coefficient reflected the magnitude of association between the intervention and the dependent outcome. All tests were two-sided, and $\mathrm{p} \leq 0.05$ was considered statistically significant. Analyses were performed using SPSS V.26. The study was approved by the institutional review board and ethics committee at both sites.

\section{RESULTS}

Figure 1 is a flow chart showing the study selection process to identify eligible visits with acute uncomplicated cystitis in the study period. After applying our prespecified exclusion criteria, our final sample included 932 visits representing 812 unique patients. Of these 932 visits, 546 were made at the intervention clinic and 386 at the control clinic. Table 3 presents the characteristics of patients at intervention and control clinics. Patients with uncomplicated cystitis visiting the control clinic were slightly younger than those in the intervention clinic ( 46.8 years versus 49 years). In both clinics, most patients were Caucasian $(62.5 \%$ and $54.9 \%$, respectively), followed by black, Asian and Hispanic patients. No significant differences were observed for race/ethnicity or Charlson Comorbidity Index.

\section{Guideline-adherent antibiotic regimen}

Table 4 summarises the proportion of guideline-adherent antibiotic regimens in each study period by study clinic. The overall proportion of guideline-adherent regimens increased both in the intervention and control clinics. For the baseline, interview and intervention periods, respectively, these values were $33.2 \%, 40.9 \%$ and $66.9 \%$ for the intervention clinic and $5.3 \%, 10.3 \%$ and $17.0 \%$ for the control clinic (table 4). The proportion of guidelineconcordant prescriptions at baseline was higher in the intervention site $(33.2 \%)$ than in the control site $(5.3 \%)$. Using the difference-in-differences analysis, the estimated net change between the intervention and baseline periods that is attributable to the intervention is 22 percentage points (table 4).

Multivariable log-binomial regression analysis of guideline-adherent regimen demonstrated a significant interaction between study clinic and study period $(p=0.01)$, showing that the increase in guideline adherence was greater in the intervention site. All RRs derived from the regression analysis including the interaction term are presented in table 5 . At the intervention site, the probability of prescribing a guideline-adherent regimen for uncomplicated cystitis was 12.7 times higher in the intervention period compared with the baseline period of the control clinic (RR 12.7 (95\% CI 6.4 to 25.2)) and 3.9 times higher compared with the intervention period of the control clinic (RR 3.9, 95\% CI 2.4 to 6.3). At the control site, the probability of prescribing a guideline-adherent regimen was 3.2 times higher in the intervention period compared with the baseline period (RR 3.2, 95\% CI 1.4

Table 4 Guideline-adherent treatment regimen during baseline, interview and intervention periods

Intervention clinic Control clinic

\begin{tabular}{|c|c|c|c|c|c|c|c|}
\hline Characteristic & Baseline & Interviews & Intervention & Baseline & Interviews & Intervention & $\begin{array}{l}\text { estimator } \\
\text { (p value) }\end{array}$ \\
\hline $\begin{array}{l}\text { Proportion† of guideline- } \\
\text { adherent regimens and } \\
95 \% \mathrm{Cl}\end{array}$ & $\begin{array}{l}33.2 \\
(26.9 \text { to } 39.9)\end{array}$ & $\begin{array}{l}40.9 \\
\text { (33.9 to } 48.2)\end{array}$ & $\begin{array}{l}66.9 \\
(58.4 \text { to } 74.6)\end{array}$ & $\begin{array}{l}5.3 \\
(2.3 \text { to } 10.1)\end{array}$ & $\begin{array}{l}10.3 \\
(5.9 \text { to } 16.4)\end{array}$ & $\begin{array}{l}17.0 \\
(9.9 \text { to } 26.6)\end{array}$ & $22(0.01) \ddagger$ \\
\hline
\end{tabular}

Baseline period was from July 2016 to March 2017, interviews from April 2017 to March 2018 and intervention from April 2018 to February 2019. *Including nitrofurantoin for 5 days or trimethoprim-sulfamethoxazole for 3 days.

†Proportion of guideline-adherent regimens was calculated by dividing guideline-adherent regimens by the total number of antibiotic prescriptions. $\ddagger$ The difference-in-differences estimator is calculated by subtracting the change in proportion of guideline-adherent regimens between the intervention and baseline periods of the control site (17.0\%-5.3\%) from the change in proportion of guideline-adherent regimens between the intervention and baseline periods of the intervention site $(66.9 \%-33.2 \%=33.7 \%)$, which is equal to $33.7 \%-11.7 \%=22 \%$. The $p$ value refers to the interaction term between study clinic (intervention and control) and study period (baseline, interviews and intervention) in the log-binomial regression analysis, implying that the increase in guideline adherence was significantly greater in the intervention site compared with the control site. 
Table 5 Effect of the intervention on the guideline adherence for uncomplicated cystitis $(n=932)$

Study period

Relative risk $(95 \% \mathrm{Cl})^{*}$

\begin{tabular}{lllc}
\cline { 2 - 4 } Site & Baseline & Interviews & Intervention \\
\hline Control site & 1 (reference) & $1.952(0.853$ to 4.465$)$ & $3.239(1.431$ to 7.331$)$ \\
Intervention site & $6.304(3.128$ to 12.704$)$ & $7.777(3.880$ to 15.590$)$ & 12.712 (6.411 to 25.206) \\
\hline
\end{tabular}

${ }^{\star}$ Relative risks are derived from the interaction between study site and study period (log-binomial regression model); adjusted for patient age.

to 7.3). The risk difference (absolute risk reduction) and $95 \% \mathrm{CI}$ of guideline adherence for uncomplicated cystitis between the intervention $(66.9 \%)$ and control $(17.0 \%)$ sites were $49.9 \%$ (38.8-60.9).

\section{DISCUSSION}

In this difference-in-differences study, we implemented a multifaceted stewardship intervention that targeted inappropriate antibiotic choice and duration of treatment. An increased proportion of guideline-adherent prescriptions was observed in the intervention period of both intervention and control sites. However, in the difference-in-differences analysis, the intervention site had a significantly larger increase in adherence than the control site.

The audit and feedback stewardship intervention was successful for UTI treatment in emergency departments $^{22} 33$ and acute ${ }^{21} 23$ and long-term care. ${ }^{21}$ In this study, we applied the audit and feedback intervention to primary care settings, where antibiotic stewardship is urgently needed. ${ }^{2}{ }^{34}$ Our intervention was based on a treatment algorithm derived from the IDSA guidelines and AAFP recommendations on management of uncomplicated UTI. ${ }^{20}{ }^{25}$ Our qualitative study showed that differentiating uncomplicated cystitis from other UTI syndromes is a challenge for providers ${ }^{28}$; therefore, providing a set of diagnostic criteria for uncomplicated cystitis was important. This treatment algorithm describes steps that providers should take when encountered with a patient with UTI-relevant symptoms. We used this algorithm as a starting point to provide personalised, interactive, one-on-one feedback with providers to improve their capacity to distinguish between uncomplicated and complicated UTI and treat UTI appropriately. The content was individualised for each recipient, and specific information about the correct solution was included to maximise feedback effectiveness. ${ }^{30}$ Besides improvements in the clinical outcomes, we also observed that the intervention was received positively by feedback recipients.

Positive impacts of antibiotic stewardship interventions in the inpatient setting and emergency department have been well described. ${ }^{21-23} 33$ In contrast, fewer studies describe successful implementation of UTI antibiotic stewardship strategies in the US outpatient setting, where an estimated $80 \%$ of antibiotic use occurs..$^{35}$ A previous study in a family medicine setting used a decision support tool embedded in the EMR to improve guideline adherence for uncomplicated UTI. ${ }^{36}$ However, the utilisation of the tool clinic-wide was only $29 \%$. Our study, one of the first to use audit and feedback in family medicine, suggests that audit and feedback is an effective approach to antibiotic stewardship in outpatient primary care clinics, although it is labour intensive.

Our study has some limitations. First, we observed improved outcomes not only in the intervention site but also in control site, which may be due to spillover effect from the providers in the intervention site to their colleagues at the control site. The clinics are in the same geographical area, and the providers from both clinics have regularly scheduled faculty meetings. Another reason for improved outcomes at the intervention and control site may be the release of FDA warnings against fluoroquinolones in the intervention period, which may have contributed to avoidance of fluoroquinolones and increased adherence to the guidelines. However, in our previous interrupted time series analysis in the same setting, the 2016 FDA boxed warning against fluoroquinolone use for simple infections was not associated with a significant reduction in the rate of fluoroquinolone prescriptions for UTI. ${ }^{5}$ Second, we assessed the choice of antibiotics and duration of therapy as these aspects of the regimen had low concordance in our previous database study in the same clinics. ${ }^{9}$ We did not assess dose or frequency of antibiotics. Third, our study was conducted only at two clinics within the same healthcare system, and certain modifications might be needed for other healthcare systems such as public healthcare system. The proportion of guideline-adherent prescriptions at baseline was higher in the intervention site. This may be explained by a lower number of visits with uncomplicated cystitis in the baseline period in the control site, so the baseline difference between the clinics may be an artefact of small sample size. Local prescribing culture and social norms may have also contributed to the baseline differences in the outcome between the clinics; culture has been previously shown to lead to differences in antibiotic prescribing in other studies. ${ }^{37}{ }^{38}$ However, different levels of outcome at baseline are acceptable for difference-in-differences analysis. ${ }^{39}$ There is no requirement that guideline-concordant prescriptions should be similar at baseline,${ }^{39}$ and our data meet the parallel trend requirement for difference-indifferences analysis. Fourth, we did not perform a formal evaluation of implementation fidelity. Fifth, our quasiexperimental study was not randomised, and we did not 
evaluate sustainability of the intervention. Perhaps most importantly, we noted that providing individualised audit and feedback is time-consuming and presents sustainability challenges.

\section{CONCLUSION}

In this difference-in-differences study, we demonstrated that our multifaceted stewardship intervention can improve the proportion of primary care clinic visits for UTI in which women receive the right drug with the right duration, a fundamental aspect of antibiotic stewardship. Future dissemination of this intervention to additional primary care clinics may incorporate a component of computerised decision support built around our UTI treatment algorithm, in addition to case-based audit and feedback, to facilitate scale-up and sustainability.

Contributors LG, RZ and BT conceived and designed the study. LG wrote the first draft of the paper. LG, GG and MG extracted the data from the institutional database and performed analysis. MS, MH, FK, MZ and RA contributed to the conception, design of the study and interpretation of data. All authors contributed intellectual content, edited the manuscript and approved the final version for submission.

Funding This investigator-initiated research study was funded by Zambon Pharmaceuticals. No award/grant number.

Disclaimer The sponsor did not participate in the study design, data collection, analysis, interpretation or preparation or submission of this report.

Competing interests LG reports grants from the US National Institutes of Health, Agency for Healthcare Research and Quality and Veterans Affairs Health Services Research and Development, outside the submitted work. BT reports grants from the US National Institutes of Health, Agency for Healthcare Research and Quality, Centers for Disease Control and Prevention and Veterans Affairs Health Services Research and Development, outside the submitted work.

Patient consent for publication Not required.

Ethics approval This study was approved by the Baylor College of Medicine Institutional Review Board (IRB). The IRB protocol ID for this study is H-38265.

Provenance and peer review Not commissioned; externally peer reviewed.

Data availability statement All data relevant to the study are included in the article or uploaded as supplemental information. Deidentified participant data.

Open access This is an open access article distributed in accordance with the Creative Commons Attribution Non Commercial (CC BY-NC 4.0) license, which permits others to distribute, remix, adapt, build upon this work non-commercially, and license their derivative works on different terms, provided the original work is properly cited, appropriate credit is given, any changes made indicated, and the use is non-commercial. See: http://creativecommons.org/licenses/by-nc/4.0/.

\section{REFERENCES}

1 Centers for Disease Control and Prevention. Antibiotic resistance threats in the United States. U.S. Department of Health and Human Services., 2019. https://www.cdc.gov/drugresistance/pdf/threatsreport/2019-ar-threats-report-508.pdf

2 The Joint Commission. Antimicrobial stewardship in ambulatory health care. Available: https://www.jointcommission.org/standards/ r3-report/r3-report-issue-23-antimicrobial-stewardship-inambulatory-health-care/

3 Meeker D, Linder JA, Fox CR, et al. Effect of behavioral interventions on inappropriate antibiotic prescribing among primary care practices: a randomized clinical trial. JAMA 2016;315:562-70.

4 Drekonja DM, Filice GA, Greer N, et al. Antimicrobial stewardship in outpatient settings: a systematic review. Infect Control Hosp Epidemiol 2015;36:142-52.

5 Bratsman A, Mathias K, Laubscher R, et al. Outpatient fluoroquinolone prescribing patterns before and after US FDA boxed warning. Pharmacoepidemiol Drug Saf 2020;29:701-7.
6 Kobayashi M, Shapiro DJ, Hersh AL, et al. Outpatient antibiotic prescribing practices for uncomplicated urinary tract infection in women in the United States, 2002-2011. Open Forum Infect Dis 2016;3:ofw159.

7 Durkin MJ, Keller M, Butler AM, et al. An assessment of inappropriate antibiotic use and guideline adherence for uncomplicated urinary tract infections. Open Forum Infect Dis 2018;5:ofy198.

8 Kabbani S, Palms D, Bartoces M, et al. Outpatient antibiotic prescribing for older adults in the United States: 2011 to 2014. J Am Geriatr Soc 2018;66:1998-2002.

9 Grigoryan L, Zoorob R, Wang $\mathrm{H}$, et al. Low concordance with guidelines for treatment of acute cystitis in primary care. Open Forum Infect Dis 2015;2:ofv159.

10 Giancola SE, Higginbotham JM, Sutter DE, et al. Improvement in adherence to antibiotic duration of therapy recommendations for uncomplicated cystitis: a quasi-experimental study. Fam Pract 2020;37:242-7

11 Denes E, Prouzergue J, Ducroix-Roubertou S, et al. Antibiotic prescription by general practitioners for urinary tract infections in outpatients. Eur J Clin Microbiol Infect Dis 2012;31:3079-83.

12 Vellinga A, Cormican M, Hanahoe B, et al. Antimicrobial management and appropriateness of treatment of urinary tract infection in general practice in Ireland. BMC Fam Pract 2011;12:108.

13 Kahan NR, Chinitz DP, Kahan E. Physician adherence to recommendations for duration of empiric antibiotic treatment for uncomplicated urinary tract infection in women: a national drug utilization analysis. Pharmacoepidemiol Drug Saf 2004;13:239-42.

14 Petty NK, Ben Zakour NL, Stanton-Cook M, et al. Global dissemination of a multidrug resistant Escherichia coli clone. Proc Natl Acad Sci U S A 2014;111:5694-9.

15 Cowart K, Worley M, Rouby NE, et al. Evaluation of FDA Boxed warning on prescribing patterns of fluoroquinolones for uncomplicated urinary tract infections. Ann Pharmacother 2019;53:1192-9.

16 U.S. Food and Drug Administration. FDA advises restricting fluoroquinolone antibiotic use for certain uncomplicated infections; warns about disabling side effects that can occur together, 2016. Available: https://www.fda.gov/downloads/Drugs/DrugSafety/ UCM500591.pdf

17 U.S. Food and Drug Administration. FDA updates warnings for fluoroquinolone antibiotics on risks of mental health and low blood sugar adverse reactions. Available: https://www.fda.gov/newsevents/ newsroom/pressannouncements/ucm612995.htm

18 U.S. Food and Drug Administration. FDA warns about increased risk of ruptures or tears in the aorta blood vessel with fluoroquinolone antibiotics in certain patients, 2018. Available: https://www.fda.gov/ Drugs/DrugSafety/ucm628753.htm

19 Tandan M, Cormican M, Vellinga A. Adverse events of fluoroquinolones vs. other antimicrobials prescribed in primary care: a systematic review and meta-analysis of randomized controlled trials. Int J Antimicrob Agents 2018;52:529-40.

20 Gupta K, Hooton TM, Naber KG, et al. International clinical practice guidelines for the treatment of acute uncomplicated cystitis and pyelonephritis in women: a 2010 update by the infectious diseases Society of America and the European Society for microbiology and infectious diseases. Clin Infect Dis 2011;52:e103-20.

21 Trautner BW, Grigoryan L, Petersen NJ, et al. Effectiveness of an antimicrobial stewardship approach for urinary catheter-associated asymptomatic bacteriuria. JAMA Intern Med 2015;175:1120-7.

22 Hecker MT, Fox CJ, Son AH, et al. Effect of a stewardship intervention on adherence to uncomplicated cystitis and pyelonephritis guidelines in an emergency department setting. PLoS One 2014;9:e87899.

23 Leis JA, Palmay L, Elligsen M, et al. Lessons from audit and feedback of hospitalized patients with bacteriuria. Am J Infect Control 2014;42:1136-7.

24 Tandan M, Burns K, Murphy H, et al. Antimicrobial prescribing and infections in long-term care facilities (LTCF): a multilevel analysis of the HALT 2016 study, Ireland, 2017. Euro Surveill 2018;23.

25 Colgan R, Williams M. Diagnosis and treatment of acute uncomplicated cystitis. Am Fam Physician 2011;84:771-6.

26 Germanos G, Light P, Zoorob R, et al. Validating use of electronic health data to identify patients with urinary tract infections in outpatient settings. Antibiotics 2020;9. doi:10.3390/ antibiotics9090536. [Epub ahead of print: 2508 2020].

27 Grigoryan L, Trautner BW, Gupta K. Diagnosis and management of urinary tract infections in the outpatient setting: a review. JAMA 2014;312:1677-84

28 Grigoryan L, Nash S, Zoorob R, et al. Qualitative analysis of primary care provider prescribing decisions for urinary tract infections. 
Antibiotics 2019;8. doi:10.3390/antibiotics8020084. [Epub ahead of print: 1906 2019].

29 Kluger AN, DeNisi A. The effects of feedback interventions on performance: a historical review, a meta-analysis, and a preliminary feedback intervention theory. Psychol Bull 1996;119:254-84.

30 Hysong SJ, Kell HJ, Petersen LA, et al. Theory-based and evidencebased design of audit and feedback programmes: examples from two clinical intervention studies. BMJ Qual Saf 2017;26:323-34.

31 Davis D, O'Brien MA, Freemantle N, et al. Impact of formal continuing medical education: do conferences, workshops, rounds, and other traditional continuing education activities change physician behavior or health care outcomes? JAMA 1999;282:867-74.

32 Livesey EA, Noon JM. Implementing guidelines: what works. Arch Dis Child Educ Pract Ed 2007;92:ep129-34.

33 Jorgensen SCJ, Yeung SL, Zurayk M, et al. Leveraging antimicrobial stewardship in the emergency department to improve the quality of urinary tract infection management and outcomes. Open Forum Infect Dis 2018;5:ofy101.

34 Fleming-Dutra KE, Hersh AL, Shapiro DJ, et al. Prevalence of inappropriate antibiotic prescriptions among US ambulatory care visits, 2010-2011. JAMA 2016;315:1864-73.
35 Centers for Disease Control and Prevention. Measuring outpatient antibiotic prescribing. Key U.S. Statistics. Available: https://www. cdc.gov/antibiotic-use/community/programs-measurement/ measuring-antibiotic-prescribing.html\#statistics

36 Eudaley ST, Mihm AE, Higdon R, et al. Development and implementation of a clinical decision support tool for treatment of uncomplicated urinary tract infections in a family medicine resident clinic. J Am Pharm Assoc 2019;59:579-85.

37 Brown KA, Daneman N, Schwartz KL, et al. The Urine-culturing cascade: variation in nursing home urine culturing and association with antibiotic use and Clostridiodes difficile infection. Clin Infect Dis 2020;70:1620-7.

38 Ratajczak M, Gold N, Hailstone S, et al. The effectiveness of repeating a social norm feedback intervention to high prescribers of antibiotics in general practice: a national regression discontinuity design. J Antimicrob Chemother 2019;74:3603-10.

39 Yoon J. Natural experiments and Difference-in-Differences. VA Health Services Research \& Development, 2019. https://www.hsrd. research.va.gov/for_researchers/cyber_seminars/archives/3553notes.pdf 